Pacific Journal of Mathematics

MEROMORPHIC MINIMAL SURFACES 


\title{
MEROMORPHIC MINIMAL SURFACES
}

\author{
E. F. Beckenbach and G. A. Hutchison
}

Meromorphic minimal surfaces are defined in this paper, and some of their differential-geometric properties are noted. The first fundamental theorem of Nevanlinna for meromorphic functions of a complex variable is extended so as to apply to these surfaces, as is the Ahlfors-Shimizu spherical version of this theorem. For these results, the classical proximity and enumerative functions of complex-variable theory are generalized, and a new visibility function is introduced. Convexity properties of some of these functions are established.

For plane meromorphic maps, the visibility function vanishes at all points on the plane but is positive at all other points of space. In general, in the present development, the sum of the enumerative function and the visibility function corresponds to the enumerative function in the classical theory.

Let a surface $S$ be given by

$$
S: x_{j}=x_{j}(u, v), \quad j=1,2,3 .
$$

Then $S$ is said to be given in terms of isothermal parameters $(u, v)$ if and only if the representation (1) is such that

$$
E=G=\lambda(u, v), \quad F=0,
$$

where

$$
E=\sum_{j=1}^{3}\left(\frac{\partial x_{j}}{\partial u}\right)^{2}, \quad F=\sum_{j=1}^{3}\left(\frac{\partial x_{j}}{\partial u}\right)\left(\frac{\partial x_{j}}{\partial v}\right), \quad G=\sum_{j=1}^{3}\left(\frac{\partial x_{j}}{\partial v}\right)^{2} .
$$

Such an isothermal representation is conformal, or angle-preserving, except at points where $\lambda(u, v)=0$.

According to a theorem of Weierstrass [13, p. 27], a necessary and sufficient condition that a surface $S$, given in terms of isothermal parameters, be minimal is that the coordinate functions be harmonic, that is, that for all $(u, v) \in D$ the functions $x_{j}(u, v), j=1,2,3$, satisfy the equation

$$
\Delta x_{j}(u, v)=0,
$$

where $\Delta$ denotes the Laplace operator,

$$
\Delta=\frac{\partial^{2}}{\partial u^{2}}+\frac{\partial^{2}}{\partial v^{2}}
$$

Then in any simply connected part of $D$, the functions given by (1) 
are the real parts of analytic functions of a complex variable,

$$
x_{j}=\mathscr{R} f_{j}(w), \quad w=u+i v,
$$

and (2) is equivalent to

$$
\sum_{j=1}^{3}\left[\frac{d f_{j}(w)}{d w}\right]^{2}=0
$$

If, in an isothermal representation (1) of a minimal surface $S$, one of the coordinate functions is identically zero, say $x_{3}(u, v) \equiv 0$, then the map lies on a plane, and either

$$
x_{1}(u, v)+i x_{2}(u, v) \quad \text { or } \quad x_{2}(u, v)+i x_{1}(u, v)
$$

is an analytic function of the complex variable $w=u+i v$. Then $x_{1}(u, v)$ and $x_{2}(u, v)$ are said to form a couple of conjugate harmonic functions. By analogy, the coordinate functions (1) of any minimal surface $S$ in isothermal representation are called a triple of conjugate harmonic functions [7]. The generalization to $\mu$-tuples of conjugate harmonic functions $x_{j}(u, v), j=1,2, \cdots, \mu$, as isothermal coordinate functions of a minimal surface $S$ in $\mu$-dimensional Euclidean space, is rather direct and will not be pursued further in this paper. ${ }^{1}$

The analogy here indicated between analytic functions of a complex variable and isothermal representations of minimal surfaces has often been noted, and since the time of Weierstrass it has served as a guiding principle in the study of minimal surfaces. It is the purpose of the present paper, as announced earlier [6], to pursue this analogy in the direction of the classical Nevanlinna theory [10] of meromorphic functions of a complex variable. Applications [4] to rational minimal surfaces and a generalization [2,3] of the second fundamental theorem of Nevanlinna to meromorphic minimal surfaces will appear elsewhere.

2. Meromorphic minimal surfaces. Let the real-valued function $x(u, v)$ be harmonic for $(u, v)$ in a deleted circular neighborhood $\mathscr{U}_{s}^{*}\left(P_{0}\right)$ of a point $P_{0}:\left(u_{0}, v_{0}\right)$, that is, for $(u, v)$ satisfying

$$
0<\left(u-u_{0}\right)^{2}+\left(v-v_{0}\right)^{2}<\varepsilon^{2} .
$$

Then $x(u, v)$ can be represented [12] in $\mathscr{U}_{c}^{*}\left(P_{0}\right)$ by a series of the form

$$
x(u, v)=c \log r+\sum_{k=-\infty}^{\infty} r^{k}\left(a_{k} \cos k \theta+b_{k} \sin k \theta\right),
$$

where $(r, \theta)$ are polar coordinates with pole $P_{0}$ :

1 By request, the results of this paper will be summarized elsewhere for minimal surfaces in $\mu$-dimensional Euclidean space, $\mu \geqq 2$. 


$$
u-u_{0}=r \cos \theta, \quad v-v_{0}=r \sin \theta .
$$

The constant $b_{0}$ is arbitrary; throughout this paper, we shall assume that it has been assigned the value 0 ,

$$
b_{0}=0 \text {. }
$$

Otherwise, the constants $c, a_{k}(k=0, \pm 1, \pm 2, \cdots)$, and $b_{k}(k= \pm 1$, $\pm 2, \cdots)$ are uniquely determined by the function $x(u, v)$.

We then have, for $\omega=w-w_{0}=(u+i v)-\left(u_{0}+i v_{0}\right)$,

$$
x(u, v)=\mathscr{R}[c \log \omega+f(\omega)], \quad \omega=r(\cos \theta+i \sin \theta),
$$

where

$$
f(\omega)=\sum_{k=-\infty}^{\infty}\left(a_{k}-i b_{k}\right) \omega^{k}
$$

is an analytic function of $\omega$ in $\mathscr{U}_{\varepsilon}^{*}\left(P_{0}\right)$.

By (7), three such functions,

$$
\begin{aligned}
x_{j}(u, v)= & c_{j} \log r \\
& +\sum_{k=-\infty}^{\infty} r_{k}\left(a_{j, k} \cos k \theta+b_{j, k} \sin k \theta\right) \\
= & \mathscr{R}\left[c_{j} \log \omega+f_{j}(\omega)\right], \quad j=1,2,3,
\end{aligned}
$$

harmonic in $\mathscr{U}_{\varepsilon}^{*}\left(P_{0}\right)$, are a triple of conjugate harmonic functions there if and only if

$$
\sum_{j=1}^{3}\left[c_{j} \omega^{-1}+\frac{d f_{j}(\omega)}{d \omega}\right]^{2}=0
$$

Now

$$
f_{j}(\omega)=\sum_{k=-\infty}^{\infty}\left(a_{j, k}-i b_{j, k}\right) \omega^{k}
$$

so that

$$
c_{j} \omega^{-1}+\frac{d f_{j}(\omega)}{d \omega}=\sum_{k=-\infty}^{\infty}\left(\alpha_{j, k}-i \beta_{j, k}\right) \omega^{n-}
$$

where for $j=1,2,3$ we have

$$
\alpha_{j, 0}=c_{j}, \quad \beta_{j, 0}=0
$$

and

$$
\alpha_{j, k}=k a_{j, k}, \quad \beta_{j, k}=k b_{j, k}, \quad k= \pm 1, \pm 2, \cdots .
$$

By (11) and (12), then, the functions (10) are a triple of conjugate harmonic functions in $\mathscr{U}_{\varepsilon}^{*}\left(P_{0}\right)$ if and only if 


$$
\sum_{j=1}^{3} \sum_{k=-\infty}^{\infty} \sum_{l=-\infty}^{\infty}\left(\alpha_{j, l}-i \beta_{j, l}\right)\left(\alpha_{j, k-l}-i \beta_{j, k-l}\right) \omega^{k-2} \equiv 0 .
$$

Accordingly, the functions (10) are a triple of conjugate harmonic functions in $\mathscr{U}_{\varepsilon}^{*}\left(P_{0}\right)$ if and only if

$$
\sum_{l=-\infty}^{\infty} \sum_{j=1}^{3}\left(\alpha_{j, l}-i \beta_{j, l}\right)\left(\alpha_{j, k-l}-i \beta_{j, k-l}\right)=0
$$

for all $k, k=0, \pm 1, \pm 2, \cdots$, that is, if and only if

$$
\sum_{l=-\infty}^{\infty} \sum_{j=1}^{3}\left(\alpha_{j, l} \alpha_{j, k-l}-\beta_{j, l} \beta_{j, k-l}\right)=0
$$

and

$$
\sum_{l=-\infty}^{\infty} \sum_{j=1}^{3}\left(\alpha_{j, l} \beta_{j, k-l}+\alpha_{j, k-l} \beta_{j, l}\right)=0
$$

for all $k, k=0, \pm 1, \pm 2, \cdots$.

Condition (16) is equivalent to

$$
\sum_{l=-\infty}^{\infty} \sum_{j=1}^{3} \alpha_{j, l} \beta_{j, k-l}=0,
$$

so that the functions (10) are a triple of conjugate harmonic functions in $\mathscr{U}_{\varepsilon}^{*}\left(P_{0}\right)$ if and only if (15) and (17) hold for all $k, k=0, \pm 1, \pm 2, \cdots$.

In terms of the original coefficients $c_{j}, a_{j, k}$, and $b_{j, k}$, by (13) and (14) the relation (15) can be written (cf. [5]) as

$$
2 k \sum_{j=1}^{3} a_{j, k} c_{j}+\sum_{l=-\infty}^{\infty} l(k-l) \sum_{j=1}^{3}\left(a_{j, l} a_{j, k-l}-b_{j, l} b_{j, k-l}\right)=0
$$

for $k= \pm 1, \pm 2, \cdots$, and as

$$
\sum_{j=1}^{3} c_{j}^{2}-\sum_{l=-\infty}^{\infty} l^{2} \sum_{j=1}^{3}\left(a_{j, l} a_{j,-l}-b_{j, l} b_{j,-l}\right)=0
$$

for $k=0$, and (17) can be written as

$$
k \sum_{j=1}^{3} b_{j, k} c_{j}+\sum_{l=-\infty}^{\infty} l(k-l) \sum_{j=1}^{3} a_{j, l} b_{j, k-l}=0
$$

for $k=0, \pm 1, \pm 2, \cdots$.

Thus, (18), (19), and (20) are necessary and sufficient conditions. for the functions (10) to be a triple of conjugate harmonic functions.

If for some $\varepsilon>0$, the functions (10) are a triple of conjugate harmonic functions in $\mathscr{U}_{*}^{*}\left(P_{0}\right)$, that is, if the functions (10) are the coordinate functions of a minimal surface $S$ in isothermal representation for $(u, v) \in \mathscr{U}_{\varepsilon}^{*}\left(P_{0}\right)$, then [unless $P_{0}$ turns out to be a regular point of $S$ (see p. 21)], we shall say that $P_{0}$ is an isolated singular point of $S$. 
If $S$ has an isolated singular point at $P_{0}$, and for an infinitude of negative indices $l$ we have

$$
\sum_{j=1}^{3}\left(a_{j, l}^{2}+b_{j, l}^{2}\right) \neq 0,
$$

then we say that the singularity of $S$ at $P_{0}$ is essential; otherwise, we say that it is nonessential.

If $S$ has a nonessential isolated singularity at $P_{0}$, and the lowest index $l=t$ for which (21) holds is negative, then we say that $S$ has a pole of order $|t|$ at $P_{0}$. By definition, then, the poles of $S$ are isolated.

We note by (19) that if (21) does not hold for any negative value of $l$, that is, if for $j=1,2,3$ we have

$$
a_{j, l}=b_{j, l}=0, \quad l=-1,-2, \cdots,
$$

then

$$
\sum_{j=1}^{3} c_{j}^{2}=0,
$$

or $c_{1}=c_{2}=c_{3}=0$. Hence, a minimal surface given in isothermal representation by functions $x_{j}(u, v)$ cannot have an isolated singularity that is merely logarithmic.

If (21) does not hold for any $l<0$, then we say that $S$ has a removable singularity at $P_{0}$. In this case, we adjoin to $S$ the point

$$
\boldsymbol{a}_{0}=\left(a_{1,0}, a_{2,0}, a_{3,0}\right)
$$

corresponding to $P_{0}$, if indeed this correspondence was not already given in the definition of $S$. Then the functions (10) determine an isothermal map of the neighborhood $\mathscr{C}_{\varepsilon}\left(P_{0}\right)$, that is, of the set of values $(u, v)$ satisfying

$$
\left(u-u_{0}\right)^{2}+\left(v-v_{0}\right)^{2}<\varepsilon^{2},
$$

onto the (extended) surface, which we again denote by $S$. We then say that $S$ is regular at $P_{0}$.

If $S$ is regular at $P_{0}$, then either each $x_{j}(u, v)$ satisfies

$$
x_{j}(u, v) \equiv a_{j, 0}, \quad j=1,2,3,
$$

and $S$ reduces to a point, or there is a lowest positive index $l=t$ for which (21) holds. In the former case, we say that $S$ is a constant minimal surface. In the latter case, we say that $S$ has an $\boldsymbol{a}_{0}$-point of order $t$ at $P_{0}$; in particular, if $a_{0}=0=(0,0,0)$ then we say that $S$ has a zero of order $t$ at $P_{0}$.

If $S$ has a pole or order $-t>0$ or an $a_{0}$-point of order $t>0$ at 
$P_{0}$, then for $k=2 t,(18)$ and $(20)$ reduce respectively to

$$
t^{2} \sum_{j=1}^{3}\left(a_{j, t}^{2}-b_{j, t}^{2}\right)=0 \text { and } t^{2} \sum_{j=1}^{3} a_{j, t} b_{j, t}=0,
$$

so that, since

$$
\begin{gathered}
\sum_{j=1}^{3}\left(a_{j, t}^{2}+b_{j, t}^{2}\right) \neq 0, \\
\sum_{j=1}^{3} a_{j, t}^{2}=\sum_{j=1}^{3} b_{j, t}^{2} \neq 0, \quad \sum_{j=1}^{3} a_{j, t} b_{j, t}=0 .
\end{gathered}
$$

In what follows, we shall frequently use the notation

$$
o(\psi(r)) \text { or } O(\psi(r))
$$

to indicate a function (not always the same function) $\varphi(r, \theta)$ such that, uniformly with respect to $\theta$, we have

$$
\lim _{r \rightarrow 0} \frac{\varphi(r, \theta)}{\psi(r)}=0 \text { or } \varlimsup_{r \rightarrow 0}\left|\frac{\varphi(r, \theta)}{\psi(r)}\right|<+\infty,
$$

respectively.

If $S$ has a pole of order $-t>0$ at $P_{0}$, then from (10) and (24) we obtain

$$
\begin{aligned}
\sum_{j=1}^{3}\left[x_{j}(u, v)\right]^{2}= & r^{2 t}\left(\sum_{j=1}^{3} a_{j, t}^{2} \cos ^{2} t \theta\right. \\
& \left.+2 \sum_{j=1}^{3} a_{j, t} b_{j, t} \cos t \theta \sin t \theta+\sum_{j=1}^{3} b_{j, t}^{2} \sin ^{2} t \theta\right)+o\left(r^{2 t}\right) \\
= & r^{2 t} \sum_{j=1}^{3} a_{j, t}^{2}+o\left(r^{2 t}\right) .
\end{aligned}
$$

Similarly, if $S$ has an $a_{0}$-point of order $t>0$ at $P_{0}$, then

$$
\sum_{j=1}^{3}\left[x_{j}(u, v)-a_{j, 0}\right]^{2}=r^{2 t} \sum_{j=1}^{3} a_{j, t}^{2}+o\left(r^{2 t}\right) .
$$

By (24) and (26) we thus see that if $S$ does not reduce to a point, then not only the poles but also the finite a-points of $S$ are isolated [5].

In analogy with complex-variable theory, for the present development we extend Euclidean 3-space by postulating a single ideal point at $\infty$. In this space, the transformation

$$
x_{j}^{*}=\frac{x_{j}}{\sum_{q=1}^{3} x_{q}^{2}}, \quad j=1,2,3,
$$

effects an inversion in the unit sphere with center at the origin, and the transformation is isothermal (see $\S 6$, below). If $S$ has a pole of order $-t>0$ at $P_{0}$, then the surface 


$$
S^{*}: x_{j}=x_{j}^{*}(u, v)=\frac{x_{j}(u, v)}{\sum_{q=1}^{3}\left[x_{q}(u, v)\right]^{2}}, \quad j=1,2,3,
$$

has a zero of order $|t|$ at $P_{0}$. The surface $S^{*}$ will not ordinarily be a minimal surface; for example, if $S$ is a plane not passing through the origin, then $S^{*}$ will be a sphere. Since the transformation is isothermal, however, we say that angles between curves on $S^{*}$ at the origin correspond to angles of the same measure on $S$ at $\infty$.

Suppose that $S$ has a pole of order $-t>0$ at $P_{0}:\left(u_{0}, v_{0}\right)$, let $P_{1}:\left(u_{1}, v_{1}\right)$ and $P_{2}\left(u_{2}, v_{2}\right)$ be nearby points at which $S$ does not have a zero, with

$$
\left(u_{j}-u_{0}, v_{j}-v_{0}\right)=\left(r_{j} \cos \theta_{j}, r_{j} \sin \theta_{j}\right), \quad j=1,2,
$$

and consider the vectors from $P_{0}$ to $P_{1}$ and from $P_{0}$ to $P_{2}$. An angle from the first of these to the second has measure $\theta_{2}-\theta_{1}$. The corresponding space vectors joining points on $S^{*}$ meet at an angle $\Theta, 0 \leqq$ $\Theta \leqq \pi$, which, by (25), satisfies

$$
\begin{aligned}
\cos \Theta & =\frac{\sum_{j=1}^{3}\left[x_{j}^{*}\left(u_{1}, v_{1}\right)\right]\left[x_{j}^{*}\left(u_{2}, v_{2}\right)\right]}{\left\{\sum_{j=1}^{3}\left[x_{j}^{*}\left(u_{1}, v_{1}\right)\right]^{2}\right\}^{\frac{1}{2}}\left\{\sum_{j=1}^{3}\left[x_{j}^{*}\left(u_{2}, v_{2}\right)\right]^{2}\right\}^{\frac{1}{2}}} \\
& =\frac{\sum_{j=1}^{3}\left[x_{j}\left(u_{1}, v_{1}\right)\right]\left[x_{j}\left(u_{2}, v_{2}\right)\right]}{\left\{\sum_{j=1}^{3}\left[x_{j}\left(u_{1}, v_{1}\right)\right]^{2}\right\}^{\frac{1}{2}}\left\{\sum_{j=1}^{3}\left[x_{j}\left(u_{2}, v_{2}\right)\right]^{2}\right\}^{\frac{1}{2}}} \\
& =\frac{\sum_{j=1}^{3} r_{1}^{t}\left(a_{j, t} \cos t \theta_{1}+b_{j, t} \sin t \theta_{1}\right) r_{2}^{t}\left(a_{j, t} \cos t \theta_{2}+b_{j, t} \sin t \theta_{2}\right)+o\left(r_{1}^{t} r_{2}^{t}\right)}{\left[r_{1}^{2 t} \sum_{j=1}^{3} a_{j, t}^{2}+o\left(r_{1}^{2 t}\right)\right]^{\frac{1}{2}}\left[r_{2}^{2 t} \sum_{j=1}^{3} a_{j, t}^{2}+o\left(r_{2}^{2 t}\right)\right]^{\frac{1}{2}}} .
\end{aligned}
$$

By (24), this reduces to

$$
\begin{aligned}
\cos \Theta & =\frac{\left(\sum_{j=1}^{3} a_{j, t}^{2}\right)\left(\cos t \theta_{1} \cos t \theta_{2}+\sin t \theta_{1} \sin t \theta_{2}\right)+o(1)}{\sum_{j=1}^{3} a_{j, t}^{2}+o(1)} \\
& =\cos t\left(\theta_{2}-\theta_{1}\right)+o(1),
\end{aligned}
$$

so that if $\theta_{2}-\theta_{1}$ has a limit $\theta_{0}$ as $r_{1} \rightarrow 0$ and $r_{2} \rightarrow 0$,

$$
\lim _{\substack{r_{1} \rightarrow 0 \\ r_{2} \rightarrow 0}}\left(\theta_{2}-\theta_{1}\right)=\theta_{0} \text {, }
$$

then

$$
\lim _{\substack{r_{1} \rightarrow 0 \\ r_{2} \rightarrow 0}} \cos \Theta=\cos t \theta_{0} .
$$


Hence the magnitudes of angles at $P_{0}$ are multiplied by $|t|$ in the map on $S^{*}$ at the origin. Therefore, by the convention given above, they are multiplied by $|t|$ in the map on $S$ at $\infty$.

Similarly, by (24) and (26), if $S$ has an $a$-point of order $t>0$ at $P_{0}$, then the magnitudes of angles at $P_{0}$ are multiplied by $t$ in the map on $S$ at $\boldsymbol{a}$.

If, except for poles, $S$ is a regular minimal surface given in isothermal representation by $(1)$ for $(u, v)$ in a finite domain $D$, then we say that $S$ is a meromorphic minimal surface for $(u, v)$ in $D$. In particular, if $D$ is the entire finite plane, then we say simply that $S$ is a meromorphic minimal surface. If $D$ is the entire finite plane and $S$ has no poles in $D$, then we say that $S$ is an entire minimal surface.

For example, the functions

$$
\begin{aligned}
& x_{1}=\mathscr{R}\left(\frac{1}{w}+w\right)=\left(\frac{1}{r}+r\right) \cos \theta, \\
& x_{2}=\mathscr{R} i\left(\frac{1}{w}-w\right)=\left(\frac{1}{r}+r\right) \sin \theta, \\
& x_{3}=\mathscr{R}(2 \log w)=2 \log r, \quad w=r(\cos \theta+i \sin \theta),
\end{aligned}
$$

are the coordinate functions of a meromorphic minimal surface (actually a catenoid) in isothermal representation. Its single pole in the finite plane is at the origin and is of order 1.

The minimal surface of Enneper [11, p. 221] is given in isothermal representation by

$$
\begin{aligned}
& x_{1}=\mathscr{R}\left(3 w-w^{3}\right)=3 r \cos \theta-r^{3} \cos 3 \theta, \\
& x_{2}=\mathscr{R} i\left(3 w+w^{3}\right)=-3 r \sin \theta-r^{3} \sin 3 \theta, \\
& x_{3}=\mathscr{R}\left(3 w^{2}\right)=3 r^{2} \cos 2 \theta, \quad w=r(\cos \theta+i \sin \theta) .
\end{aligned}
$$

This is an entire minimal surface. Its single zero is at the origin and is of order 1.

The relations

$$
\begin{aligned}
& x_{1}=\mathscr{R}\left(\log w-\frac{1}{2} w^{2}\right)=\log r-\frac{1}{2} r^{2} \cos 2 \theta, \\
& x_{2}=\mathscr{R} i\left(\log w+\frac{1}{2} w^{2}\right)=-i \theta-\frac{1}{2} r^{2} \sin 2 \theta, \\
& x_{3}=\mathscr{R}(2 w)=2 r \cos \theta, \quad w=r(\cos \theta+i \sin \theta),
\end{aligned}
$$

give an isothermal representation of a minimal surface with a singularity of a different sort at the origin. The second of the relations (29) is not a (single-valued) function of $w=u+i v$, however, so that this surface is not included in the class of surfaces presently under consideration.

The subclass of meromorphic minimal surfaces (1) for which the 
$c_{j}$ are restricted to have the value 0 in the representation (10) for each $P_{0}$ is somewhat more tractable than the unrestricted class. The restriction is not needed for the validity of the results of this paper, however, and accordingly we shall not make it here.

3. Formulas. In this section, we shall develop some formulas that will be needed later. These formulas are concerned with the differential geometry of meromorphic minimal surfaces.

Let the functions (10) be the coordinate functions of a nonconstant meromorphic minimal surface $S$ in isothermal representation in $\mathscr{C}_{\varepsilon}^{*}\left(P_{0}\right)$, and let $\tau$ denote the lowest index $l$ for which we have

$$
\sum_{j=1}^{3} a_{j, l}^{2} \neq 0 \text {. }
$$

By (9) and (24), $\tau$ is then also the lowest index for which (21) holds. Equation (10) can accordingly be written as

$$
\begin{aligned}
x_{j}(u, v) & =c_{j} \log r+\sum_{k=\tau}^{\infty} r^{k}\left(a_{j, k} \cos k \theta+b_{j, k} \sin k \theta\right) \\
& =\mathscr{R}\left[c_{j} \log \omega+f_{j}(\omega)\right], \quad j=1,2,3,
\end{aligned}
$$

where

$$
\sum_{j=1}^{3} a_{j, \tau}^{2} \neq 0
$$

and

$$
f_{j}(\omega)=\sum_{k=\tau}^{\infty}\left(a_{j, k}-i b_{j, k}\right) \omega^{k} .
$$

If $\tau=0$, let $t$ denote the lowest positive index $l$ for which (21) holds; if $\tau \neq 0$, let $t=\tau$. Then $t \geqq \tau$, with inequality if and only if $\tau=0$. Recalling that (22) holds if $\tau \geqq 0$, we see that:

If $\tau<0$, then $t=\tau$ and $S$ has a pole of order $-t$ at $P_{0}$.

If $\tau=0$, then $t>\tau$ and $S$ has an $a_{0}$-point $\left(a_{0} \neq 0\right)$ of order $t$ at $P_{0}$.

If $\tau>0$, then $t=\tau$ and $S$ has a zero of order $t$ at $P_{0}$.

If $\tau<0$, then $t=\tau$, so that (25) can be written as

$$
\sum_{j=1}^{3}\left[x_{j}(u, v)\right]^{2}=r^{2 \tau} \sum_{j=1}^{3} a_{j, \tau}^{2}+o\left(r^{2 \tau}\right) .
$$

If $\tau=0$ then, because of (22), (34) follows from (31) by direct computation. If $\tau>0$, then again $t=\tau$; since now $\boldsymbol{a}_{0}=0,(26)$ can be written as (34) in this case. Hence (34) holds in all cases. 
From (31) and the definition of $t$, by differentiating we obtain

$$
\frac{\partial x_{j}}{\partial u}-i \frac{\partial x_{j}}{\partial v}=\frac{c_{j}}{\omega}+\sum_{k=t}^{\infty} k\left(a_{j, k}-i b_{j, k}\right) \omega^{k-1} .
$$

Equating real parts, and equating imaginary parts, in (35), we therefore have

$$
\begin{aligned}
\frac{\partial x_{j}}{\partial u}= & \frac{c_{j}}{r} \cos \theta \\
& +\sum_{k=t}^{\infty} k r^{k-1}\left[a_{j, k} \cos (k-1) \theta+b_{j, k} \sin (k-1) \theta\right], \\
\frac{\partial x_{j}}{\partial v}= & \frac{c_{j}}{r} \sin \theta \\
& +\sum_{k=t}^{\infty} k r^{k-1}\left[b_{j, k} \cos (k-1) \theta-a_{j, k} \sin (k-1) \theta\right] .
\end{aligned}
$$

From (2), (3), (24), and (36), we obtain

$$
\lambda(u, v)=t^{2} r^{2 t-2} \sum_{j=1}^{3} a_{j, t}^{2}+O\left(r^{2 t-1}\right) .
$$

It follows from (24) and (38) (cf. [5]) that for a nonconstant meromorphic minimal surface $S$ given in terms of isothermal parameters $(u, v)$, the zeros and infinities of the area-deformation ratio $\lambda(u, v)$ are isolated.

At points where $\lambda(u, v) \neq 0$ and $\lambda(u, v) \neq \infty, S$ has a tangent plane. The direction cosines $X_{j}(u, v)$ of its normal are given [11, p. 147] by

$$
x_{j}(u, v)=\frac{\frac{\partial x_{k}(u, v)}{\partial u} \frac{\partial x_{l}(u, v)}{\partial v}-\frac{\partial x_{l}(u, v)}{\partial u} \frac{\partial x_{k}(u, v)}{\partial v}}{\lambda(u, v)},
$$

where $j, k, l=1,2,3$ in cyclic order. For the functions (31), let $\varepsilon>0$ be so small that $\lambda(u, v) \neq 0$ and $\lambda(u, v) \neq \infty$ in $\mathscr{Q}_{\varepsilon}^{*}\left(P_{0}\right)$. Then for $(u, v) \in \mathscr{U}_{*}^{*}\left(P_{0}\right)$, from (36), (37), (38), and (39) we obtain, by a computation,

$$
\begin{aligned}
X_{j}(u, v) & =\frac{t^{2} r^{2 t-2}\left(a_{k, t} b_{l, t}-a_{l, t} b_{k, t}\right)+O\left(r^{2 t-1}\right)}{t^{2} r^{2 t-2} \sum_{q=1}^{3} a_{q, t}^{2}+O\left(r^{2 t-1}\right)} \\
& =\frac{a_{k, t} b_{l, t}-a_{l, t} b_{k, t}}{\sum_{q=1}^{3} a_{q, t}^{2}}+O(r) .
\end{aligned}
$$

By (40), we see that 


$$
\lim _{r \rightarrow 0} X_{j}(u, v)=\frac{a_{k, t} b_{l, t}-a_{l, t} b_{k, t}}{\sum_{q=1}^{3} a_{q, t}^{2}},
$$

$j, k, l=1,2,3$ in cyclic order, even if $\lambda\left(u_{0}, v_{0}\right)=0$ or $\lambda\left(u_{0}, v_{0}\right)=\infty$. We take this limiting value (41) as the definition of $X_{j}\left(u_{0}, v_{0}\right)$ if $\lambda\left(u_{0}, v_{0}\right)=$ 0 or $\lambda\left(u_{0}, v_{0}\right)=\infty$. With this extended definition of the functions $X_{j}(u, v)$, we see by (24) that a nonconstant meromorphic minimal surface $S$ given in terms of isothermic parameters $(u, v)$ has a continuous unit normal vector function

$$
\boldsymbol{X}(u, v)=\left(X_{1}(u, v), X_{2}(u, v), X_{3}(u, v)\right)
$$

throughout the domain in which $S$ is meromorphic.

The next formula we shall develop is fundamental for the present investigation. It is an expression [7] for the Laplacian of the logarithm of the distance function

$$
\left.\left\{\sum_{j=1}^{3} x_{j}(u, v)\right]^{2}\right\}^{\frac{1}{2}}
$$

for a nonconstant meromorphic minimal surface given in isothermal representation.

For the isothermal coordinate functions (31) of a nonconstant meromorphic minimal surface $S$, let $\varepsilon>0$ be so small that in $\mathscr{U}_{\varepsilon}^{*}\left(P_{0}\right)$ the distance function (42) and the area-deformation ratio $\lambda(u, v)$ have no zeros or infinities. They might or might not vanish or be infinite at $P_{0}$.

Using vector notation, for $(u, v) \in \mathscr{U}_{\varepsilon}^{*}\left(P_{0}\right)$ we obtain, by a computation,

$$
\begin{aligned}
& \Delta \log (\boldsymbol{x} \cdot \boldsymbol{x})^{\frac{1}{2}} \\
& \quad=\frac{(\boldsymbol{x} \cdot \boldsymbol{x})\left(\boldsymbol{x} \cdot \Delta \boldsymbol{x}+\boldsymbol{x}_{u} \cdot \boldsymbol{x}_{u}+\boldsymbol{x}_{v} \cdot \boldsymbol{x}_{v}\right)-2\left\lceil\left(\boldsymbol{x} \cdot \boldsymbol{x}_{u}\right)^{2}+\left(\boldsymbol{x} \cdot \boldsymbol{x}_{v}\right)^{2}\right\rceil}{(\boldsymbol{x} \cdot \boldsymbol{x})^{2}},
\end{aligned}
$$

where the subscripts indicate partial differentiation. By (2) and (3) we have

$$
\boldsymbol{x}_{u} \cdot \boldsymbol{x}_{u}=\boldsymbol{x}_{v} \cdot \boldsymbol{x}_{v}=\lambda(u, v),
$$

and from (4) we obtain

$$
\Delta \boldsymbol{x}=0
$$

Hence (43) reduces to

$$
\Delta \log (x \cdot x)^{\frac{1}{2}}=\frac{2\left[(x \cdot x) \lambda-\left(x \cdot x_{n}\right)^{2}-\left(x \cdot x_{v}\right)^{2}\right]}{(x \cdot x)^{2}} .
$$

Since $\lambda(u, v) \neq 0$ in $\mathscr{U}_{\varepsilon}^{*}\left(P_{0}\right), \boldsymbol{x}_{u}$ and $\boldsymbol{x}_{v}$ are nonnull vectors there. 
Further, these vectors lie in the tangent plane to $S$ and therefore are perpendicular to the unit normal vector $X(u, v)$; and since $F=0$, they are perpendicular to each other. Accordingly, for any $(u, v) \in \mathscr{U}_{s}^{*}\left(P_{0}\right)$, there are scalars $\alpha, \beta, \gamma$ such that

$$
\boldsymbol{x}=\alpha \boldsymbol{x}_{u}+\beta \boldsymbol{x}_{v}+\gamma \boldsymbol{X} .
$$

From (45), we obtain

$$
\begin{aligned}
& \boldsymbol{x} \cdot \boldsymbol{x}=\alpha^{2} \lambda+\beta^{2} \lambda+\gamma^{2}, \\
& \boldsymbol{x} \cdot \boldsymbol{x}_{u}=\alpha \lambda, \\
& \boldsymbol{x} \cdot \boldsymbol{x}_{v}=\beta \lambda, \\
& \boldsymbol{x} \cdot \boldsymbol{X}=\gamma .
\end{aligned}
$$

Hence (44) can be written as

$$
\Delta \log (\boldsymbol{x} \cdot \boldsymbol{x})^{\frac{1}{2}}=\frac{2\left(\alpha^{2} \lambda^{2}+\beta^{2} \lambda^{2}+\gamma^{2} \lambda-\alpha^{2} \lambda^{2}-\beta^{2} \lambda^{2}\right)}{(\boldsymbol{x} \cdot \boldsymbol{x})^{2}}=\frac{2 \gamma^{2} \lambda}{(\boldsymbol{x} \cdot \boldsymbol{x})^{2}},
$$

or, finally, the fundamental formula

$$
\Delta \log (x \cdot x)^{\frac{1}{2}}=\frac{2(x \cdot X)^{2} \lambda}{(x \cdot x)^{2}} .
$$

In obtaining an estimate of the behavior of the right-hand member of (46) as $r \rightarrow 0$, we can use the expressions (34) and (38) for $\boldsymbol{x} \cdot \boldsymbol{x}$ and $\lambda$, respectively. For $\boldsymbol{x} \cdot \boldsymbol{X}$, by (31) and (40) we have

$$
\boldsymbol{x} \cdot \boldsymbol{X}=\frac{\boldsymbol{r}^{\tau} \sum_{j, k, l}\left(a_{j, \tau} \cos \tau \theta+b_{j, \tau} \sin \tau \theta\right)\left(a_{k, t} b_{l, t}-a_{l, t} b_{k, t}\right)}{\sum_{q=1}^{3} a_{q, t}^{2}}+o\left(r^{\tau}\right),
$$

where $j, k, l=1,2,3$ in cyclic order in the sum in the numerator.

If $\tau=0$, then by (24), (32), (34), (38), and (47) we have

$$
\begin{aligned}
\lim _{r \rightarrow 0}(\boldsymbol{x} \cdot \boldsymbol{x})^{2} & =\left(\sum_{j=1}^{3} a_{j, 0}^{2}\right)^{2}>0, \\
\lim _{r \rightarrow 0} \lambda & =t^{2} \sum_{j=1}^{3} a_{j, t}^{2} \lim _{r \rightarrow 0} r^{2 t-2} \geqq 0,
\end{aligned}
$$

with equality if and only if $t>1$, and

$$
\lim _{r \rightarrow 0}(\boldsymbol{x} \cdot \boldsymbol{X})^{2}=\left[\frac{\sum_{j, k, l} a_{j, 0}\left(a_{k, t} b_{l, t}-a_{l, t} b_{k, t}\right)}{\sum_{q=1}^{3} a_{q, t}^{2}}\right]^{2}>0 .
$$

Since each of these three limits exists and is finite, and the limit of $(\boldsymbol{x} \cdot \boldsymbol{x})^{2}$ is not 0 , it follows from (46) that $\Delta \log (\boldsymbol{x} \cdot \boldsymbol{x})^{\frac{1}{2}}$ is continuous at $\left(u_{0}, v_{0}\right)$ in this case. 
If $\tau \neq 0$, then $t=\tau$, and the sum in the numerator of the first term in the right-hand member of (47) vanishes identically. We then have, by (31) and (40),

$$
\boldsymbol{x} \cdot \boldsymbol{X}=O\left(r^{\tau+1}\right)
$$

unless

$$
\tau=-1 \text { and } \sum_{j=1}^{3} c_{j}^{2} \neq 0
$$

In the exceptional case (49), we have

$$
\boldsymbol{x} \cdot \boldsymbol{X}=O(\log r) \text {. }
$$

If (48) holds, then from (34), (38), (46), and (48) we get

$$
\Delta \log (\boldsymbol{x} \cdot \boldsymbol{x})^{\frac{1}{2}}=\frac{O\left(r^{2 t-2}\right)\left[O\left(r^{t+1}\right)\right]^{2}}{\left[r^{2 t} \sum_{j=1}^{3} a_{j, t}^{2}+o\left(r^{2 t}\right)\right]^{2}}=O\left(r^{2 t-2+2 t+2-4 t}\right)=O(1),
$$

so that $\Delta \log (x \cdot x)^{\frac{1}{2}}$ is bounded in the neighborhood of $P_{0}$. Notice, however, that it is not necessarily continuous at $P_{0}$. For example, for the minimal surface of Enneper (28), we have

$$
\Delta \log (x \cdot x)^{\frac{1}{2}}=2 \cos ^{2} 2 \theta+o(1),
$$

so that the limiting behavior of $\Delta \log (\boldsymbol{x} \cdot \boldsymbol{x})^{\frac{1}{2}}$ depends on the limiting behavior of $\theta$. Thus if $\theta \rightarrow 0$ as $r \rightarrow 0$ then $\Delta \log (x \cdot x)^{\frac{1}{2}} \rightarrow 2$, but if $\theta \rightarrow \pi / 4$ as $r \rightarrow 0$ then $\Delta \log (\boldsymbol{x} \cdot \boldsymbol{x})^{\frac{1}{2}} \rightarrow 0$.

In the exceptional case (49), in place of (51) we have, by (32) and (50),

$$
\begin{aligned}
\Delta \log (\boldsymbol{x} \cdot \boldsymbol{x})^{\frac{1}{2}} & =\frac{O\left(r^{-4}\right)[O(\log r)]^{2}}{\left[r^{-2} \sum_{j=1}^{3} a_{j,-1}^{2}+o\left(r^{-2}\right)\right]^{2}} \\
& =O\left[r^{-4+4}(\log r)^{2}\right]=O\left[(\log r)^{2}\right] .
\end{aligned}
$$

For example, for the catenoid (27) we have

$$
\Delta \log (x \cdot x)^{\frac{1}{2}}=8(\log r)^{2}[1+o(1)] .
$$

Thus in the exceptional case (49), we see that $\Delta \log (x \cdot x)^{\frac{1}{2}}$ becomes infinite as $r \rightarrow 0$. As we shall see in the next section, however, it does not become infinite too rapidly for the applications we shall be making.

4. An application of Green's theorem. Let $w=u+i v$; let $A_{r}\left(w_{0}\right)$ denote the closed circular disc $\left|w-w_{0}\right| \leqq r$; and let $\partial A_{r}\left(w_{0}\right)$ denote the boundary, $\left|w-w_{0}\right|=r$, of $A_{r}\left(w_{0}\right)$.

Let the functions (1) be the coordinate functions of a nonconstant 
meromorphic minimal surface $S$ in isothermal representation for $(u, v)$ in a finite domain $D$, and for a given fixed $R>0$ let $A_{R}(0)$ be contained in $D$.

If $S$ has a zero at the origin, let $s_{0}$ denote the order of this zero; otherwise, let $s_{0}=0$. Similarly, if $S$ has a pole at the origin, let $n_{0}$ denote the order of this pole, and otherwise let $n_{0}=0$. Of course, at least one of $s_{0}$ and $n_{0}$ must be equal to 0 , and both might be equal to 0 .

Let $s_{0}$ be denoted by $n(0,0 ; S)$, and $n_{0}$ by $n(0, \infty ; S)$. Then by (34) and the definition of $\tau$, if $\tau_{0}$ is the value of $\tau$, and $t_{0}$ that of $t$, for the functions (31) representing $S$ when $P_{0}$ is the origin, we have

$$
\boldsymbol{x} \cdot \boldsymbol{x}=r^{2 \tau_{0}} \sum_{j=1}^{3} a_{j, \tau_{0}}^{2}+o\left(r^{2 \tau_{0}}\right), \quad r=|w|,
$$

with

$$
\sum_{j=1}^{3} \alpha_{j, \tau_{0}}^{2} \neq 0
$$

and

$$
\tau_{0}=s_{0}-n_{0}=n(0,0 ; S)-n(0, \infty ; S) .
$$

For any $\rho, 0<\rho \leqq R$, there can be only a finite number of zeros and poles of $S$ in $A_{\rho}(0)$, since the zeros and poles of a nonconstant meromorphic minimal surface are isolated. In the punctured disc $0<|w| \leqq \rho$, let the zeros of $S$ be at the points

$$
w=z_{1}, z_{2}, \cdots, z_{k}, \quad k=k(\rho) \geqq 0,
$$

with

$$
0<\left|z_{1}\right| \leqq\left|z_{2}\right| \leqq \cdots \leqq\left|z_{k}\right| \leqq \rho,
$$

and let the poles be at

$$
w=p_{1}, p_{2}, \cdots, p_{l}, \quad l=l(\rho) \geqq 0,
$$

with

$$
0<\left|p_{1}\right| \leqq\left|p_{2}\right| \leqq \cdots \leqq\left|p_{l}\right| \leqq \rho .
$$

Let the orders of these zeros and poles be, respectively,

$$
s_{1}, s_{2}, \cdots, s_{k} \text { and } n_{1}, n_{2}, \cdots, n_{l} \text {, }
$$

and denote the sum of the orders of the zeros and poles of $S$ in $A_{\rho}(0)$ by $n(\rho, 0 ; S)$ and $n(\rho, \infty ; S)$, respectively:

$$
\begin{aligned}
& n(\rho, 0 ; S)=s_{0}+s_{1}+\cdots+s_{k}, \\
& n(\rho, \infty ; S)=n_{0}+n_{1}+\cdots+n_{l} .
\end{aligned}
$$


Then $n(\rho, 0 ; S) \geqq 0$, with $s_{j}>0$ for $j=1,2, \cdots, k$, and similarly $n(\rho, \infty ; S) \geqq 0$, with $n_{q}>0$ for $q=1,2, \cdots, l$.

Now let $\rho, 0<\rho \leqq R$, be chosen so that $S$ does not have a zero or pole on $\partial A_{\rho}(0)$. Since there are only a finite number of zeros and poles of $S$ in $A_{\rho}(0)$, we can choose $r>0$ so small that the $k+l+1$ closed circular discs $A_{r}(0), A_{r}\left(z_{j}\right), j=1,2, \cdots, k$, and $A_{r}\left(p_{q}\right), \quad q=$ $1,2, \cdots, l$, are disjoint from one another and interior to $A_{\rho}(0)$. Let $\Omega_{r}$ denote the domain interior to the circle $\partial A_{\rho}(0)$ and exterior to the circles $\partial A_{r}(0), \partial A_{r}\left(z_{j}\right)$, and $\partial A_{r}\left(p_{q}\right), j=1, \cdots, k$ and $q=1,2, \cdots, l$.

In $\Omega_{r}$, the function

$$
g(u, v)=\log (\boldsymbol{x} \cdot \boldsymbol{x})^{\frac{1}{2}}
$$

has continuous derivatives of all orders. Hence we can apply Green's theorem to $g(u, v)$ in $\Omega_{r}$ :

$$
\int_{\partial \Omega_{r}} \frac{\partial g}{\partial \nu} d s=\iint_{\Omega_{r}} \Delta g(u, v) d A
$$

where $\nu$ refers to the normal directed outwardly from $\Omega_{r}$.

By the definition of $\Omega_{r}$, we have

$$
\begin{aligned}
\int_{\partial \Omega_{r}} \frac{\partial g(u, v)}{d \nu} d s= & \int_{\partial_{A_{\rho}}(0)} \frac{\partial g}{\partial \rho} \rho d \theta-\int_{\partial_{A_{r}}(0)} \frac{\partial g}{\partial r} r d \theta \\
& -\sum_{j=1}^{k} \int_{\partial_{A_{r}\left(z_{j}\right)}} \frac{\partial g}{\partial r} r d \theta-\sum_{q=1}^{l} \int_{\partial_{A_{\boldsymbol{r}}\left(p_{q}\right)}} \frac{\partial g}{\partial r} r d \theta .
\end{aligned}
$$

From (31), (53), and (55), by a computation we find that on $\partial A_{r}(0)$ we have

$$
\frac{\partial g}{\partial r}=\frac{\tau_{0}}{r}+o\left(r^{-1}\right)=\frac{s_{0}-n_{0}}{r}+o\left(r^{-1}\right)
$$

so that

$$
\int_{\partial_{A_{\boldsymbol{r}}(0)}} \frac{\partial g}{\partial r} r d \theta=2 \pi \tau_{0}+o(1)=2 \pi\left(s_{0}-n_{0}\right)+o(1) .
$$

Similarly, on the $\partial A_{r}\left(z_{j}\right)$ and $\partial A_{r}\left(p_{q}\right)$ we have, respectively,

$$
\frac{\partial g}{\partial r}=\frac{s_{j}}{r}+o\left(r^{-1}\right)
$$

and

$$
\frac{\partial g}{\partial r}=\frac{-n_{q}}{r}+o\left(r^{-1}\right)
$$

so that 


$$
\int_{\partial_{A_{r}\left(z_{j}\right)}} \frac{\partial g}{\partial r} r d \theta=2 \pi s_{j}+o(1)
$$

and

$$
\int_{\partial A_{r}\left(p_{q}\right)} \frac{\partial g}{\partial r} r d \theta=-2 \pi n_{q}+o(1) .
$$

By (56), (57), and (60), we accordingly have

$$
\begin{aligned}
\int_{\partial \Omega_{r}} \frac{\partial g(u, v)}{d \nu} d s= & \int_{\partial A_{\rho}(0)} \frac{\partial g}{\partial \rho} \rho d \theta-2 \pi \sum_{j=0}^{k} s_{j} \\
& +2 \pi \sum_{q=0}^{l} n_{q}+o(1) \\
= & \int_{\partial A_{\rho}(0)} \frac{\partial g}{\partial \rho} \rho d \theta-2 \pi n(\rho, 0 ; S) \\
& +2 \pi n(\rho, \infty ; S)+o(1),
\end{aligned}
$$

whence

$$
\begin{aligned}
& \lim _{r \rightarrow 0} \int_{\partial \Omega_{r}} \frac{\partial g(u, v)}{d \nu} d s \\
& \quad=\int_{\partial A_{\rho}(0)} \frac{\partial g}{\partial \rho} \rho d \theta-2 \pi n(\rho, 0 ; S)+2 \pi n(\rho, \infty ; S) .
\end{aligned}
$$

By (51) and (52), for any $A_{r}(w) \subset D$ we have

$$
\iint_{A_{r}(w)} \Delta g(u, v) d A=\int_{0}^{2 \pi} \int_{0}^{r} O(1) \sigma d \sigma d \theta
$$

or at worst, in the exceptional case (49),

$$
\iint_{A_{r}(w)} \Delta g(u, v) d A=\int_{0}^{2 \pi} O\left[(\log \sigma)^{2}\right] \sigma d \sigma d \theta
$$

Since

$$
\sigma O(1)=o(1) \text { and } \sigma O\left[(\log \sigma)^{2}\right]=o(1)
$$

in either case we have

$$
\lim _{r \rightarrow 0} \iint_{A_{r}(w)} \Delta g(u, v) d A=0 .
$$

Therefore, by the definition of $\Omega_{r}$,

$$
\lim _{r \rightarrow 0} \iint_{\Omega_{r}} \Delta g(u, v) d A=\iint_{A_{\rho}(0)} \Delta g(u, v) d A .
$$

From (59), (61), and (62), we obtain 


$$
\int_{\partial A \rho^{(0)}} \frac{\partial g}{\partial \rho} \rho d \theta-2 \pi n(\rho, 0 ; S)+2 \pi n(\rho, \infty ; S)=\iint_{A_{\rho}(0)} \Delta g(u, v) d A,
$$

whence

$$
\begin{aligned}
& \frac{1}{2 \pi} \int_{\partial_{A} \rho^{(0)}} \frac{\partial g}{\partial \rho} d \theta-\frac{n(\rho, 0 ; S)-n(\rho, \infty ; S)}{\rho} \\
& \quad=\frac{1}{2 \pi \rho} \iint_{A_{\rho}(0)} \Delta g(u, v) d A .
\end{aligned}
$$

Now (63) can be written as

$$
\begin{aligned}
& \frac{1}{2 \pi} \int_{0}^{2 \pi} \frac{\partial g}{\partial \rho} d \theta-\frac{n(0,0 ; S)-n(0, \infty ; S)}{\rho}-\frac{n(\rho, 0 ; S)-n(0,0 ; S)}{\rho} \\
& \quad+\frac{n(\rho, \infty ; S)-n(0, \infty ; S)}{\rho}=\frac{1}{2 \pi \rho} \iint_{A_{\rho}(0)} \Delta g(u, v) d A
\end{aligned}
$$

Nothing that, by (55),

$$
\begin{aligned}
-\frac{n(0,0 ; S)-n(0, \infty ; S)}{\rho} & =\frac{-\tau_{0}}{\rho}=\frac{\partial}{\partial \rho} \log \rho^{-\tau_{0}} \\
& =\frac{1}{2 \pi} \int_{0}^{2 \pi} \frac{\partial}{\partial \rho} \log \rho^{-\tau_{0}} d \theta,
\end{aligned}
$$

we see from (58) that

$$
\begin{gathered}
\frac{1}{2 \pi} \int_{0}^{2 \pi} \frac{\partial g}{\partial \rho} d \theta-\frac{n(0, \boldsymbol{0} ; S)-n(0, \infty ; S)}{\rho} \\
=\frac{1}{2 \pi} \int_{0}^{2 \pi} \frac{\partial}{\partial \rho} \log \left[(\boldsymbol{x} \cdot \boldsymbol{x})^{\frac{1}{2}} \rho^{-\tau_{0}}\right] d \theta .
\end{gathered}
$$

By (65), we can therefore rewrite (64) as

$$
\begin{aligned}
& \frac{1}{2 \pi} \int_{0}^{2 \pi} \frac{\partial}{\partial \rho} \log \left[(\boldsymbol{x} \cdot \boldsymbol{x})^{\frac{1}{2}} \rho^{-\tau_{0}}\right] d \theta-\frac{n(\rho, 0 ; S)-n(0,0 ; S)}{\rho} \\
& \quad+\frac{n(\rho, \infty ; S)-n(0, \infty ; S)}{\rho}=\frac{1}{2 \pi \rho} \iint_{A_{\rho}(0)} \Delta g(u, v) d A .
\end{aligned}
$$

By (51) and (52), for any $r, 0<r \leqq R$, the right-hand member of (66) can be integrated from 0 to $r$ with respect to $\rho$. The numerators in the second and third terms on the left vanish in an interval $0 \leqq \rho \leqq r_{0}, r_{0}>0$, so these terms also can be integrated. For the first term in the left-hand member of (66), by (53) we have

$$
\begin{aligned}
& \int_{\varepsilon}^{r}\left\{\frac{1}{2 \pi} \int_{0}^{2 \pi} \frac{\partial}{\partial \rho} \log \left[(\boldsymbol{x} \cdot \boldsymbol{x})^{\frac{1}{2}} \rho^{-\tau_{0}}\right] d \theta\right\} d \rho \\
& \quad=\frac{1}{2 \pi} \int_{0}^{2 \pi}\left\{\int_{\varepsilon}^{r} \frac{\partial}{\partial \rho} \log \left[(\boldsymbol{x} \cdot \boldsymbol{x})^{\frac{1}{2}} \rho^{-\tau} 0\right] d \rho\right\} d \theta
\end{aligned}
$$




$$
\begin{aligned}
= & \left.\frac{1}{2 \pi} \int_{0}^{2 \pi} \log \left[(\boldsymbol{x} \cdot \boldsymbol{x})^{\frac{1}{2}} \rho^{-\tau_{0}}\right]\right|_{\varepsilon} ^{r} d \theta \\
= & \frac{1}{2 \pi} \int_{\partial A_{\rho}(0)} \log (\boldsymbol{x} \cdot \boldsymbol{x})^{\frac{1}{2}} d \theta-\tau_{0} \log r \\
& -\log \left(\sum_{j=1}^{3} a_{j, \tau_{0}}^{2}\right)^{\frac{1}{2}}+o(1),
\end{aligned}
$$

so that

$$
\begin{aligned}
\lim _{\varepsilon \rightarrow 0} \int_{\varepsilon}^{r}\left\{\frac{1}{2 \pi} \int_{0}^{2 \pi} \frac{\partial}{\partial \rho} \log \left[(\boldsymbol{x} \cdot \boldsymbol{x})^{\frac{1}{2}} \rho^{-\tau_{0}}\right] d \theta\right\} d \rho \\
=\frac{1}{2 \pi} \int_{\partial A_{r}(0)} \log (\boldsymbol{x} \cdot \boldsymbol{x})^{\frac{1}{2}} d \theta-n(0,0 ; S) \log r \\
\quad+n(0, \infty ; S) \log r-\log \left(\sum_{j=1}^{3} a_{j, \tau_{0}}^{2}\right)^{\frac{1}{2}} .
\end{aligned}
$$

Hence (66) yields

(67)

$$
\begin{aligned}
& \frac{1}{2 \pi} \int_{\partial_{A_{r}(0)}} \log (\boldsymbol{x} \cdot \boldsymbol{x})^{\frac{1}{2}} d \theta \\
& \quad-\int_{0}^{r} \frac{n(\rho, 0 ; S)-n(0,0 ; S)}{\rho} d \rho-n(0,0 ; S) \log r \\
& \quad+\int_{0}^{r} \frac{n(\rho, \infty ; S)-n(0, \infty ; S)}{\rho} d \rho+n(0, \infty ; S) \log r \\
& \quad-\log \left(\sum_{j=1}^{3} a_{j, \tau_{0}}^{2}\right)^{\frac{1}{2}}=\frac{1}{\pi} \int_{0}^{r}\left[\frac{1}{2 \rho} \iint_{A_{\rho}(0)} \Delta g(u, v) d A\right] d \rho .
\end{aligned}
$$

If for a nonnegative function $\varphi$ the function $\log ^{+} \varphi$ is defined by

$$
\log ^{+} \varphi=\left\{\begin{array}{ll}
\log \varphi & \text { for } \varphi \geqq 1, \\
0 & \text { for } 0 \leqq \varphi \leqq 1
\end{array},\right.
$$

then we have the identity

$$
\log \varphi=\log ^{+} \varphi-\log ^{+} \frac{1}{\varphi}
$$

and (67) can be written, by (46) and (58), as

$$
\begin{aligned}
& \frac{1}{2 \pi} \int_{\partial_{A_{r}(0)}} \log ^{+}(\boldsymbol{x} \cdot \boldsymbol{x})^{\frac{1}{2}} d \theta \\
& \quad+\int_{0}^{r} \frac{n(\rho, \infty ; S)-n(0, \infty ; S)}{\rho} d \rho+n(0, \infty ; S) \log r \\
& \quad=\frac{1}{2 \pi} \int_{\partial_{A_{r}(0)}} \log ^{+}(\boldsymbol{x} \cdot \boldsymbol{x})^{-\frac{1}{2}} d \theta
\end{aligned}
$$




$$
\begin{aligned}
& +\int_{0}^{r} \frac{n(\rho, 0 ; S)-n(0,0 ; S)}{\rho} d \rho+n(0,0 ; S) \log r \\
& +\int_{0}^{r}\left[\frac{1}{\rho} \iint_{A_{\rho}(0)} \frac{(\boldsymbol{x} \cdot \boldsymbol{X})^{2}}{\pi(\boldsymbol{x} \cdot \boldsymbol{x})^{2}} \lambda d A\right] d \rho+\log \left(\sum_{j=1}^{3} a_{j, \tau_{0}}^{2}\right)^{\frac{1}{2}}
\end{aligned}
$$

5. The Nevanlinna characteristic function. For a nonconstant meromorphic minimal surface $S$ given in isothermic representation by the functions (1), and for any given finite

$$
\boldsymbol{a}=\left(a_{1}, a_{2}, a_{3}\right)
$$

consider the surface

$$
S-a: x_{j}=x_{j}(u, v)-a_{j}, \quad j=1,2,3,
$$

for $(u, v) \in D$. This again is a nonconstant meromorphic minimal surface in isothermic representation.

Applying (68) to $S-\boldsymbol{a}$, we obtain

$$
\begin{aligned}
\frac{1}{2 \pi} \int_{\partial_{A} r^{(0)}} & \log ^{+}[(\boldsymbol{x}-\boldsymbol{a}) \cdot(\boldsymbol{x}-\boldsymbol{a})]^{\frac{1}{2}} d \theta \\
& +\int_{0}^{r} \frac{n(\rho, \infty ; S-\boldsymbol{a})-n(0, \infty ; S-\boldsymbol{a})}{\rho} d \rho \\
& +n(0, \infty ; S-\boldsymbol{a}) \log r \\
= & \frac{1}{2 \pi} \int_{\partial_{A_{r}(0)}} \log ^{+}[(\boldsymbol{x}-\boldsymbol{a}) \cdot(\boldsymbol{x}-\boldsymbol{a})]^{-\frac{1}{2}} d \theta \\
& +\int_{0}^{r} \frac{n(\rho, 0 ; S-\boldsymbol{a})-n(0,0 ; S-\boldsymbol{a})}{\rho} d \rho \\
& +n(0,0 ; S-\boldsymbol{a}) \log \boldsymbol{r} \\
& +\int_{0}^{r}\left\{\frac{1}{\rho} \iint_{A_{\rho}(0)} \frac{[(\boldsymbol{x}-\boldsymbol{a}) \cdot \boldsymbol{X}]^{2}}{\pi[(\boldsymbol{x}-\boldsymbol{a}) \cdot(\boldsymbol{x}-\boldsymbol{a})]^{2}} \lambda d A\right\} \alpha_{i} \\
& +\log \left(\sum_{j=1}^{3} a_{j, \tau_{\boldsymbol{a}}}^{2}\right)^{\frac{1}{2}},
\end{aligned}
$$

where $\tau_{\boldsymbol{a}}$ is the value of $\tau$, and $t_{a}$ that of $t_{0}$, for the functions (69), and the $a_{j, \tau}$ are leading coefficients of these functions.

Since the poles of $S$ and the poles of $S-\boldsymbol{a}$ occur at the same $(u, v)$-points, we have

$$
n(\rho, \infty ; S)=n(\rho, \infty ; S-\boldsymbol{a}) .
$$

Since the zeros of the surface $S-a$ are the $a$-points of the surface $S$, the function $n(\rho, \boldsymbol{a} ; S)$, defined by

$$
n(\rho, \boldsymbol{a} ; S)=n(\rho, 0 ; S-\boldsymbol{a}),
$$


gives an expression of the number of $a$-points of the surface $S$ in $|w| \leqq \rho$.

If the map of $A_{\rho}(0)$ on $S$ is projected from $a$ on the unit sphere with center at $\boldsymbol{a}$, then the function $h(\rho, \boldsymbol{a} ; S)$, defined by

$$
h(\rho, \boldsymbol{a} ; S)=\iint_{A_{\rho}(0)} \frac{[(\boldsymbol{x}-\boldsymbol{a}) \cdot \boldsymbol{X}]^{2}}{\pi[(\boldsymbol{x}-\boldsymbol{a}) \cdot(\boldsymbol{x}-\boldsymbol{a})]^{2}} \lambda d A,
$$

gives a measure of the area of this projection, with the element of area of the projection weighted by

$$
\frac{1}{\pi}|\cos (x-a, X)| \text {. }
$$

Thus $h(\rho, \boldsymbol{a} ; S)$ can be considered as a measure of the visibility of the surface $S$ for $|w| \leqq \rho$, as viewed from $a$.

In particular, we have

$$
h(0, \boldsymbol{a} ; S)=0 .
$$

Since

$$
\lim _{\boldsymbol{a} \rightarrow \infty} h(\rho, \boldsymbol{a} ; S)=0,
$$

we define $h(\rho, \infty ; S)$ by

$$
h(\rho, \infty ; S)=0 .
$$

By analogy with the Nevanlinna theory of meromorphic functions. of a complex variable, let us define a proximity function (Schmiegungsfunktion) for $S$ by

$$
\begin{aligned}
& m(r, \infty ; S)=\frac{1}{2 \pi} \int_{\partial_{A_{r}(0)}} \log ^{+}(\boldsymbol{x} \cdot \boldsymbol{x})^{\frac{1}{2}} d \theta, \\
& m(\boldsymbol{r}, \boldsymbol{a} ; S)=\frac{1}{2 \pi} \int_{\partial_{A_{r}(0)}} \log ^{+}[(\boldsymbol{x}-\boldsymbol{a}) \cdot(\boldsymbol{x}-\boldsymbol{a})]^{-\frac{1}{2}} d \theta,
\end{aligned}
$$

and an enumerative function (Anzahlfunktion) by

$$
\begin{aligned}
N(r, \infty ; S) & =\int_{0}^{r} \frac{n(\rho, \infty ; S)-n(0, \infty ; S)}{\rho} d \rho+n(0, \infty ; S) \log r, \\
N(r, \boldsymbol{a} ; S) & =\int_{0}^{r} \frac{n(\rho, \boldsymbol{a} ; S)-n(0, \boldsymbol{a} ; S)}{\rho} d \rho+n(0, \boldsymbol{a} ; S) \log r,
\end{aligned}
$$

for $a$ finite.

To these we now adjoin a visibility function (Sichbarkeitsfunktion), defined by

$$
\begin{aligned}
H(r, \infty ; S) & =0, \\
H(r, \boldsymbol{a} ; S) & =\int_{0}^{r} \frac{h(\rho, \boldsymbol{a} ; S)}{\rho} d \rho .
\end{aligned}
$$


By (74) and (75), the definition (78) of $H$ is quite analogous to the definition (77) of $N$, with $h$ in place of $n$.

Substituting from (76), (77), and (78) into (68) and (70), and using (71) and (72), we obtain, respectively,

$$
\begin{aligned}
& m(r, \infty ; S)+N(r, \infty ; S)+H(r, \infty ; S) \\
& \quad=m(r, 0 ; S)+N(r, 0 ; S)+H(r, 0 ; S)+\log \left(\sum_{j=1}^{3} a_{j, \tau_{0}}^{2}\right)^{\frac{1}{2}}
\end{aligned}
$$

and

$$
\begin{aligned}
& m(r, \infty, S-\boldsymbol{a})+N(r, \infty ; S)+H(r, \infty ; S) \\
& \quad=m(\boldsymbol{r}, \boldsymbol{a} ; S)+N(r, \boldsymbol{a} ; S)+H(\boldsymbol{r}, \boldsymbol{a} ; S)+\log \left(\sum_{j=1}^{3} a_{j, \tau}^{2}\right)^{\frac{1}{2}} .
\end{aligned}
$$

It is well known that for any two nonnegative numbers, say $\gamma_{1}$ and $\gamma_{2}$ with $\gamma_{1} \geqq \gamma_{2} \geqq 0$, we have

$$
\log ^{+}\left(\gamma_{1}+\gamma_{2}\right) \leqq \log ^{+} \gamma_{1}+\log ^{+} \gamma_{2}+\log 2 \text {. }
$$

To establish (81), notice that for $\gamma_{1} \geqq 1$ we have

$$
\begin{aligned}
\log ^{+}\left(\gamma_{1}+\gamma_{2}\right) & =\log \left(\gamma_{1}+\gamma_{2}\right) \leqq \log 2 \gamma_{1} \\
& =\log \gamma_{1}+\log 2 \leqq \log ^{+} \gamma_{1}+\log ^{+} \gamma_{2}+\log 2,
\end{aligned}
$$

while for $\gamma_{1}<1$ we have

$$
\log ^{+}\left(\gamma_{1}+\gamma_{2}\right) \leqq \log 2=\log ^{+} \gamma_{1}+\log ^{+} \gamma_{2}+\log 2 .
$$

Hence (81) holds in any case.

By the triangle inequality, we have

$$
[(x-a) \cdot(x-a)]^{\frac{1}{2}} \leqq(x \cdot x)^{\frac{1}{2}}+(a \cdot a)^{\frac{1}{2}}
$$

and

$$
\begin{aligned}
(\boldsymbol{x} \cdot \boldsymbol{x})^{\frac{1}{2}} & =[(\boldsymbol{x}-\boldsymbol{a}+\boldsymbol{a}) \cdot(\boldsymbol{x}-\boldsymbol{a}+\boldsymbol{a})]^{\frac{1}{2}} \\
& \leqq[(\boldsymbol{x}-\boldsymbol{a}) \cdot(\boldsymbol{x}-\boldsymbol{a})]^{\frac{1}{2}}+(\boldsymbol{a} \cdot \boldsymbol{a})^{\frac{1}{2}} .
\end{aligned}
$$

Accordingly, from (76) and (81) we obtain

$$
m(r, \infty ; S-\boldsymbol{a}) \leqq m(r, \infty ; S)+\log ^{+}(\boldsymbol{a} \cdot \boldsymbol{a})^{\frac{1}{2}}+\log 2
$$

and

$$
m(r, \infty ; S) \leqq m(r, \infty ; S-\boldsymbol{a})+\log ^{+}(\boldsymbol{a} \cdot \boldsymbol{a})^{\frac{1}{2}}+\log 2 .
$$

From (82) and (83), we have

$$
m(r, \infty ; S)-m(r, \infty ; S-\boldsymbol{a})=B(r, \boldsymbol{a} ; S),
$$

with 


$$
|B(r, \boldsymbol{a} ; S)| \leqq \log ^{+}(\boldsymbol{a} \cdot \boldsymbol{a})^{\frac{1}{2}}+\log 2 .
$$

Substituting for $m(r, \infty ; S-\boldsymbol{a})$ from (84) into (80), we get

$$
\begin{aligned}
& m(r, \infty ; S)+N(r, \infty ; S)+H(r, \infty ; S) \\
& \quad=m(r, \boldsymbol{a} ; S)+N(r, \boldsymbol{a} ; S)+H(r, \boldsymbol{a} ; S)+C(r, \boldsymbol{a} ; S),
\end{aligned}
$$

where

$$
C(r, \boldsymbol{a} ; S)=\log \left(\sum_{j=1}^{3} a_{j, \tau_{a}}^{2}\right)^{\frac{1}{2}}+B(r, \boldsymbol{a} ; S) .
$$

By (85) and (87), we have

$$
|C(r, \boldsymbol{a} ; S)| \leqq\left|\log \left(\sum_{j=1}^{3} \alpha_{j, \tau_{\boldsymbol{a}}}^{2}\right)^{\frac{1}{2}}\right|+\log ^{+}(\boldsymbol{a} \cdot \boldsymbol{a})^{\frac{1}{2}}+\log 2 .
$$

We define the total affinity of $S$ to $\boldsymbol{a}$ in $|w| \leqq r$, or the affinity function for $S$, by

$$
\mathfrak{U}(r, \boldsymbol{a} ; S)=m(r, \boldsymbol{a} ; S)+N(r, \boldsymbol{a} ; S)+H(r, \boldsymbol{a} ; S) .
$$

In particular, we call the total affinity of $S$ to $\infty$ the Nevanlinna characteristic function of $S$ and denote it by $T(r ; S)$, so that

$$
\begin{aligned}
T(r ; S) & =\mathfrak{A}(r, \infty ; S) \\
& =m(r, \infty ; S)+N(r, \infty ; S)+H(r, \infty ; S) \\
& =m(r, \infty ; S)+N(r, \infty ; S) .
\end{aligned}
$$

The first fundamental theorem of R. Nevanlinna [10] concerning meromorphic functions of a complex variable is generalized by means of the inequality (88) to meromorphic minimal surfaces:

THEOREM 1. If the functions

$$
x_{j}=x_{j}(u, v), \quad j=1,2,3,
$$

are the coordinate functions of a nonconstant meromorphic minimal surface $S$ in isothermal representation for $u^{2}+v^{2}<\infty$, then for each finite a we have

$$
T(r, S)=\mathfrak{A}(r, \boldsymbol{a} ; S)+C(r, \boldsymbol{a} ; S),
$$

where $C(r, \boldsymbol{a} ; S)$ is a bounded function of $r$ for each $\boldsymbol{a}$ :

$$
|C(r, \boldsymbol{a} ; S)| \leqq\left|\log \left(\sum_{j=1}^{3} a_{j, \tau_{2}}^{2}\right)^{\frac{1}{2}}\right|+\log ^{+}(\boldsymbol{a} \cdot \boldsymbol{a})^{\frac{1}{2}}+\log 2 .
$$

Thus $S$ has essentially the same affinity for all points $a$ in space, in the sense that for any two given points $a$ and $b$ the difference 


$$
\mathfrak{U}(r, \boldsymbol{a} ; S)-\mathfrak{U}(r, \boldsymbol{b} ; S)
$$

is a bounded function of $r$ :

$$
\begin{aligned}
& |\mathfrak{A}(r, \boldsymbol{a} ; S)-\mathfrak{A}(r, \boldsymbol{b} ; S)| \leqq\left|\log \left(\sum_{j=1}^{3} a_{j, \tau_{\boldsymbol{a}}}^{2}\right)^{\frac{1}{2}}\right| \\
& \quad+\left|\log \left(\sum_{j=1}^{3} a_{j, \tau_{\boldsymbol{b}}}^{2}\right)^{\frac{1}{2}}\right|+\log ^{+}(\boldsymbol{a} \cdot \boldsymbol{a})^{\frac{1}{2}}+\log ^{+}(\boldsymbol{b} \cdot \boldsymbol{b})^{\frac{1}{2}}+\log 4 .
\end{aligned}
$$

This is true in particular if $S$ lies on a plane (the complex-variable case) and the point $\boldsymbol{a}$, or the point $\boldsymbol{b}$, or both, are not on the plane.

6. The hyperspherical characteristic function. Because of the $\log ^{+}$function in the formulas (76), the value of the proximity function $m(r, \boldsymbol{a} ; S)$ is affected only by the portion of the map of $\partial A_{r}(0)$ on $S$ that lies at distance $\leqq 1$ from $a$ for $a$ finite, or at distance $\geqq 1$ from 0 for $\boldsymbol{a}=\infty$.

In the Ahlfors-Shimizu theory $[1,14 ; 9,15]$ for the complex-variable case, the plane of the map $S$ is projected stereographically [8, pp. 119, 120] onto a spherical surface of radius $\frac{1}{2}$, and then the chordal distance is used as a metric. In this metric, each point of the plane is at distance $\leqq 1$ from each other point of the plane, and accordingly all of the map of $\partial A_{r}(0)$ on $S$ contributes to the proximity function for each point $\boldsymbol{a}$ of the plane.

An analogous treatment can be given for isothermal maps on nonconstant meromorphic minimal surfaces.

In the four-dimensional $\left(x_{1}, x_{2}, x_{3}, x_{4}\right)$-space, let $\mathscr{S}_{0}$ be the hypersphere with center

$$
\boldsymbol{x}^{0}=\left(x_{1}^{0}, x_{2}^{0}, x_{3}^{0}, x_{4}^{0}\right)
$$

and radius $\delta_{0}$. Then, as in three-dimensional inversion, the points

$$
\boldsymbol{x}=\left(x_{1}, x_{2}, x_{3}, x_{4}\right) \text { and } \boldsymbol{x}^{\prime}=\left(x_{1}^{\prime}, x_{2}^{\prime}, x_{3}^{\prime}, x_{4}^{\prime}\right)
$$

are said to be inverses of each other with respect to $\mathscr{S}_{0}$ if and only if $\boldsymbol{x}$ and $\boldsymbol{x}^{\prime}$ are on the same ray with endpoint $\boldsymbol{x}^{0}$ and are such that

$$
\delta \delta^{\prime}=\delta_{0}^{2},
$$

where

$$
\delta=\left[\left(x-x^{0}\right) \cdot\left(x-x^{0}\right)\right]^{\frac{1}{2}} \text { and } \delta^{\prime}=\left[\left(x^{\prime}-x^{0}\right) \cdot\left(x^{\prime}-x\right)\right]^{\frac{1}{2}}
$$

are the Euclidean distances in four-dimensional space from $\boldsymbol{x}^{0}$ to $\boldsymbol{x}$ and from $\boldsymbol{x}^{0}$ to $\boldsymbol{x}^{\prime}$, respectively.

By similar triangles, then, $\boldsymbol{x}$ and $\boldsymbol{x}^{\prime}$ are inverses of each other with respect to $\mathscr{S}_{0}$ if and only if 


$$
\frac{x_{j}^{\prime}-x_{j}^{0}}{x_{j}-x_{j}^{0}}=\frac{\delta^{\prime}}{\delta}=\frac{\delta \delta^{\prime}}{\delta^{2}}=\frac{\delta_{0}^{2}}{\delta^{2}}, \quad j=1,2,3,4,
$$

or

$$
x_{j}^{\prime}-x_{j}^{0}=\frac{\delta_{0}^{2}}{\delta^{2}}\left(x_{j}-x_{j}^{0}\right), \quad j=1,2,3,4
$$

As in the three-dimensional case [8, pp. 117-120], inversion in $\mathscr{S}_{0}$ maps four-dimensional space in a one-to-one way onto itself, with $\boldsymbol{x}^{0}$ corresponding to a unique ideal point, $\infty$, at infinity. A hypersphere or hyperplane is mapped onto a hypersphere or hyperplane, according as the given hypersphere or hyperplane does not or does pass through $\boldsymbol{x}^{0}$.

Further, from (94) we obtain

$$
d x_{j}^{\prime}=\frac{\delta_{0}^{2}}{\delta^{2}} d x_{j}-\frac{2 \delta_{0}^{2}}{\delta^{3}}\left(x_{j}-x_{j}^{0}\right) d \delta,
$$

whence

$$
\begin{aligned}
d s^{\prime 2}= & d \boldsymbol{x}^{\prime} \cdot d \boldsymbol{x}^{\prime} \\
= & \frac{\delta_{0}^{4}}{\delta^{4}} d \boldsymbol{x} \cdot d \boldsymbol{x}-\frac{4 \delta_{0}^{4}}{\delta^{5}} d \delta\left(\boldsymbol{x}-\boldsymbol{x}^{0}\right) \cdot d \boldsymbol{x} \\
& +\frac{4 \delta_{0}^{4}}{\delta^{6}}(d \delta)^{2}\left(\boldsymbol{x}-\boldsymbol{x}^{0}\right) \cdot\left(\boldsymbol{x}-\boldsymbol{x}^{0}\right) \\
= & \frac{\delta_{0}^{4}}{\delta^{4}} d \boldsymbol{x} \cdot d \boldsymbol{x}=\frac{\delta_{0}^{4}}{\delta^{4}} d s^{2},
\end{aligned}
$$

or

$$
d s^{\prime}=\frac{\delta_{0}^{2}}{\delta^{2}} d s
$$

so that the transformation is an isothermal one.

For the particular choice

$$
\boldsymbol{x}^{0}=(0,0,0,1) \text { and } \delta_{0}=1
$$

(94) yields

$$
\begin{aligned}
x_{j}^{\prime} & =\frac{x_{j}}{x_{1}^{2}+x_{2}^{2}+x_{3}^{2}+\left(x_{4}-1\right)^{2}}, \quad j=1,2,3, \\
x_{4}^{\prime} & =\frac{x_{1}^{2}+x_{2}^{2}+x_{3}^{2}+x_{4}\left(x_{4}-1\right)}{x_{1}^{2}+x_{2}^{2}+x_{3}^{2}+\left(x_{4}-1\right)^{2}} .
\end{aligned}
$$

Under this inversion, the coordinates of the image of a point $\left(x_{1} x_{2}, x_{3}, 0\right)$ on the hyperplane $x_{4}=0$ are given by 
(96)

$$
\begin{aligned}
x_{j}^{\prime} & =\frac{x_{j}}{x_{1}^{2}+x_{2}^{2}+x_{3}^{2}+1} \\
& =\frac{x_{j}}{1+\boldsymbol{x} \cdot \boldsymbol{x}}, \quad j=1,2,3, \\
x_{4}^{\prime} & =\frac{x_{1}^{2}+x_{2}^{2}+x_{3}^{2}}{x_{1}^{2}+x_{2}^{2}+x_{3}^{2}+1} \\
& =\frac{\boldsymbol{x} \cdot \boldsymbol{x}}{1+\boldsymbol{x} \cdot \boldsymbol{x}} .
\end{aligned}
$$

The coordinates (96) satisfy

$$
x_{1}^{\prime 2}+x_{2}^{\prime 2}+x_{3}^{\prime 2}+\left(x_{4}^{\prime}-\frac{1}{2}\right)^{2}=\left(\frac{1}{2}\right)^{2},
$$

so that the image of $\left(x_{1}, x_{2}, x_{3}, 0\right)$ lies on the sphere $\mathscr{S}$ with center $\left(0,0,0, \frac{1}{2}\right)$ and radius $\frac{1}{2}$. In fact, the stereographic projection of the hypersphere $\mathscr{S}$ from its "north" pole $(0,0,0,1)$ onto the hyperplane $x_{4}=0$ tangent to $\mathscr{S}$ at its "south" pole $(0,0,0,0)$ coincides with the mapping of $\mathscr{S}$ onto this hyperplane under inversion in $\mathscr{S}_{0}$.

We shall henceforth call the hyperplane $x_{4}=0$ the $\left(x_{1}, x_{2}, x_{3}\right)$-space. For points

$$
\boldsymbol{x}=\left(x_{1}, x_{2}, x_{3},\right) \text { and } \boldsymbol{y}=\left(y_{1}, y_{2}, y_{3}\right)
$$

in the finite $\left(x_{1}, x_{2}, x_{3}\right)$-space, the line segment joining their images

$$
\boldsymbol{x}^{\prime}=\left(x_{1}^{\prime}, x_{2}^{\prime}, x_{3}^{\prime}, x_{4}^{\prime}\right) \text { and } \boldsymbol{y}^{\prime}=\left(y_{1}^{\prime}, y_{2}^{\prime}, y_{3}^{\prime}, y_{4}^{\prime}\right)
$$

under the inversion in $\mathscr{S}_{0}$ described above is a chord of the sphere $\mathscr{S}$. If we let $\chi(\boldsymbol{x}, \boldsymbol{y})$ denote the length of this chord,

$$
\chi(x, y)=\operatorname{distance}\left(x^{\prime}, y^{\prime}\right)=\left[\left(x^{\prime}-y^{\prime}\right) \cdot\left(x^{\prime}-y^{\prime}\right)\right]^{\frac{1}{2}}
$$

then we have

$$
0 \leqq \chi(x, y) \leqq 1
$$

From (96), we obtain

$$
\begin{aligned}
(1+ & x \cdot x)^{2}(1+y \cdot y)^{2}[\chi(x, y)]^{2} \\
= & {[(1+y \cdot y) x-(1+x \cdot x) y] \cdot[(1+y \cdot y) x-(1+x \cdot x) y] } \\
& +[(1+y \cdot y)(x \cdot x)-(1+x \cdot x)(y \cdot y)]^{2} \\
= & (1+x \cdot x)(1+y \cdot y)[(1+y \cdot y)(x \cdot x)+(1+x \cdot x)(y \cdot y) \\
& -2(x \cdot y)-2(x \cdot x)(y \cdot y)],
\end{aligned}
$$

so that

$$
\begin{aligned}
(1+x \cdot x)(1+y \cdot y)[\chi(x, y)]^{2} & =x \cdot x-2 x \cdot y+y \cdot y \\
& =(x-y) \cdot(x-y)
\end{aligned}
$$


or

$$
\chi(x, y)=\frac{[(x-y) \cdot(x-y)]^{\frac{1}{2}}}{(1+x \cdot x)^{\frac{1}{2}}(1+y \cdot y)^{\frac{1}{2}}}
$$

In the limit, as $y \rightarrow \infty$, (98) gives

$$
\chi(x, \infty)=\frac{1}{(1+x \cdot x)^{\frac{1}{2}}} .
$$

We now define a hyperspherical proximity function for $S$, analogous to (76), in terms of the chordal distance:

$$
m^{\circ}(r, \boldsymbol{a} ; S)=\frac{1}{2 \pi} \int_{\partial_{A_{r}(0)}} \log \frac{1}{\chi(\boldsymbol{x}, \boldsymbol{a})} d \theta .
$$

Notice that here the integrand, which in (76) was restricted to nonnegative values by use of the $\log ^{+}$function, takes on only nonnegative values by virtue of (97).

By (98), (99), and (100), we have

$$
\begin{aligned}
m^{\circ}(r, \infty ; S) & =\frac{1}{2 \pi} \int_{\partial_{A_{r}}(0)} \log (1+\boldsymbol{x} \cdot \boldsymbol{x})^{\frac{1}{2}} d \theta, \\
m^{\circ}(\boldsymbol{r}, \boldsymbol{a} ; S) & =\frac{1}{2 \pi} \int_{\partial_{A_{\boldsymbol{r}}(0)}} \log \frac{(1+\boldsymbol{x} \cdot \boldsymbol{x})^{\frac{1}{2}}(1+\boldsymbol{a} \cdot \boldsymbol{a})^{\frac{1}{2}}}{[(\boldsymbol{x}-\boldsymbol{a}) \cdot(\boldsymbol{x}-\boldsymbol{a})]^{\frac{1}{2}}} d \theta
\end{aligned}
$$

for $\boldsymbol{a}=\left(a_{1}, a_{2}, a_{3}\right)$ finite.

From (76), (101), and the fact that

$$
\log \varphi=\log ^{+} \varphi-\log ^{+} \frac{1}{\varphi},
$$

for $\boldsymbol{a}$ finite we obtain

$$
\begin{aligned}
m^{\circ}(\boldsymbol{r}, \boldsymbol{a} ; S)= & m^{\circ}(r, \infty ; S)+m(r, \boldsymbol{a} ; S) \\
& -m(r, \infty ; S-\boldsymbol{a})+\log (1+\boldsymbol{a} \cdot \boldsymbol{a})^{\frac{1}{2}}
\end{aligned}
$$

Substituting from (102) into (80), we get

$$
\begin{aligned}
& m^{\circ}(r, \infty ; S)+N(r, \infty ; S)+H(r, \infty ; S) \\
& \quad=m^{\circ}(\boldsymbol{r}, \boldsymbol{a} ; S)+N(r, \boldsymbol{a} ; S)+H(r, \boldsymbol{a} ; S) \\
& \quad+\log \frac{\left(\sum_{j=1}^{3} a_{j, \tau \boldsymbol{a}}^{2}\right)^{\frac{1}{2}}}{(1+\boldsymbol{a} \cdot \boldsymbol{a})^{\frac{1}{2}}} .
\end{aligned}
$$

We can write (103) as

$$
\begin{aligned}
& m^{\circ}(r, \infty ; S)+N(r, \infty ; S)+H(r, \infty ; S)+C(\infty ; S) \\
& \quad=m^{\circ}(r, \boldsymbol{a} ; S)+N(r, \boldsymbol{a} ; S)+H(r, \boldsymbol{a} ; S)+C(\boldsymbol{a} ; S),
\end{aligned}
$$


where the constants $C(\infty ; S)$ and $C(\boldsymbol{a} ; S)$ are such that in (104) both the left-hand member and the right-hand member $\rightarrow 0$ as $r \rightarrow 0$. Namely, if there is not a pole of $S$ at $r=0$ then we have

$$
\begin{aligned}
C(\infty ; S) & =\log \frac{1}{\left(1+\sum_{j=1}^{3} a_{j, 0}^{2}\right)^{\frac{1}{2}}}, \\
C(\boldsymbol{a} ; S) & =\log \frac{\left(\sum_{j=1}^{3} a_{j, \tau}^{2}\right)^{\frac{1}{2}}}{\left(1+\sum_{j=1}^{3} a_{j, 0}^{2}\right)^{\frac{1}{2}}(1+\boldsymbol{a} \cdot \boldsymbol{a})^{\frac{1}{2}}},
\end{aligned}
$$

while if there is a pole of $S$ at $r=0$ then $t_{a}=t_{0}<0$ is independent of $\boldsymbol{a}$,

$$
a_{j, \tau a}=a_{j, t_{0}}, \quad j=1,2,3,
$$

and we have

$$
\begin{gathered}
C(\infty, S)=\log \frac{1}{\left(\sum_{j=1}^{3} a_{j, t_{0}}^{2}\right)^{\frac{1}{2}}}, \\
C(\boldsymbol{a} ; S)=\log \frac{1}{(1+\boldsymbol{a} \cdot \boldsymbol{a})^{\frac{1}{2}}} .
\end{gathered}
$$

It might be noted that in complex-variable theory the equation analogous to (104) does not include the terms $H(r, \infty ; S)$ and $H(r, a ; S)$, for then these terms are identically zero. Neither does the complexvariable equation ordinarily include constant terms $C(\infty ; S)$ and $C(\boldsymbol{a} ; S)$; here, however, the distinction is only notational, for the constants are then included in the definitions either [9] of $m^{\circ}(r, \infty, S)$ and $m^{\circ}(r, \boldsymbol{a} ; S)$ or [15] of $N(r, \infty ; S)$ and $N(r, \boldsymbol{\alpha} ; S)$.

We now define the hyperspherical affinity of $S$ to $a$ in $|w| \leqq r$, or the hyperspherical affinity function for $S$, by

$$
\begin{aligned}
\mathfrak{H}^{\circ}(r, \boldsymbol{a} ; S)= & m^{\circ}(\boldsymbol{r}, \boldsymbol{a} ; S)+N(r, \boldsymbol{a} ; S) \\
& +H(\boldsymbol{r}, \boldsymbol{a} ; S)+C(\boldsymbol{a} ; S) .
\end{aligned}
$$

In particular, we call the hyperspherical affinity of $S$ to $\infty$ the hyperspherical characteristic function of $S$ and denote it by $T^{\circ}(r ; S)$ :

$$
\begin{aligned}
T^{\circ}(r ; S) & =\mathfrak{A}^{\circ}(r, \infty ; S) \\
& =m^{\circ}(r, \infty ; S)+N(r, \infty ; S)+H(r ; \infty ; S)+C(\infty ; S) .
\end{aligned}
$$

Substituting from (107) and (108) in (104), we have the following generalization of the Ahlfors-Shimizu spherical form of the first fundamental theorem of Nevanlinna to meromorphic minimal surfaces:

THEOREM 2. If the functions

$$
x_{j}=x_{j}(u, v), \quad j=1,2,3,
$$


are the coordinate functions of a nonconstant meromorphic minimal surface in isothermal representation for $u^{2}+v^{2}<\infty$, then for each finite $\boldsymbol{a}=\left(a_{1}, a_{2}, a_{3}\right)$ we have

$$
T^{\circ}(r ; S)=\mathfrak{U}^{\circ}(r, \boldsymbol{a} ; S),
$$

where $\mathfrak{U}^{\circ}(r, \boldsymbol{a} ; S)$ is the hyperspherical affinity of $S$ to $\boldsymbol{a}$, and $T^{\circ}(r ; S)=\mathfrak{A}^{\circ}(r, \infty ; S)$ is the hyperspherical characteristic function of $S$.

\section{Convexity properties. For}

$$
u^{2}+v^{2}=|w|^{2}=|u+i v|^{2}<\infty,
$$

let the functions (1) be the coordinate functions of a nonconstant meromorphic minimal surface $S$ in isothermal representation. For $a$ finite or infinite, let the $a$-points of $S$ in $0<|w| \leqq r \leqq r$ be at the points $w=w_{j}, j=1,2, \cdots, k$, of moduli $r_{1} \leqq r_{2} \leqq \cdots \leqq r_{k}$, and let the respective orders of these $a$-points be $\alpha_{j}, j=1,2, \cdots, k$. Then

$$
n(r, \boldsymbol{a} ; S)=\alpha_{0}+\alpha_{1}+\cdots+\alpha_{k},
$$

where $\alpha_{0} \geqq 0$ is the order of the $a$-point, if any, at $w=0$.

Evaluating the integral in (77), we obtain

$$
N(r, \boldsymbol{a} ; S)=\log \frac{r^{n(r, \boldsymbol{a} ; S)}}{r_{1}^{\alpha_{1}} r_{2}^{\alpha_{2}} \cdots r_{k}^{\alpha_{k}}} .
$$

From either (77) or (110), we see that $N(r, \boldsymbol{a} ; S)$ is a continuous function of $\log r$. Further, by differentiating either (77) or (110), we get

$$
\frac{d N(r, \boldsymbol{a} ; S)}{d \log r}=n(r, \boldsymbol{a} ; S)
$$

except at the points of discontinuity of $n(r, \boldsymbol{\alpha} ; S)$. Accordingly, since $n(r, \boldsymbol{a} ; S)$ is a nondecreasing, nonnegative function of $\log r$, we have the following result:

The function $N(\boldsymbol{r}, \boldsymbol{a} ; S)$ is a nondecreasing, piecewise linear, convex function of $\log r$.

Similarly, for $a$ finite, by differentiating the second equation in (78) we obtain

$$
\frac{d H(\boldsymbol{r}, \boldsymbol{a} ; S)}{d \log r}=h(r, \boldsymbol{a} ; S)
$$

By (73), we have

$$
h(r, \boldsymbol{a} ; S) \geqq 0,
$$

with equality for $r>0$ if and only if $S$ is a plane surface and $a$ lies 
in the plane. Further, by (78), we have

$$
H(r, \boldsymbol{a} ; S)>0 \text { for } r>0 \text { if } h(r, \boldsymbol{a} ; S) \not \equiv 0 .
$$

Hence we have the following result:

The function $H(r, a ; S)$ vanishes identically if $\boldsymbol{a}$ is infinite or if $S$ is a plane surface and $a$ lies in the plane. Otherwise, for $r>0, H(r, a ; S)$ is a positive, increasing, strictly convex function of $\log r$.

To determine the behavior of $T^{\circ}(r ; S)$, we integrate (109) with respect to $\boldsymbol{a}$ over the three-dimensional hyperspherical "surface" $\mathscr{S}$ and divide by the content

$$
V=2 \pi^{2}\left(\frac{1}{2}\right)^{3}=\frac{\pi^{2}}{4}
$$

of $\mathscr{S}$ to obtain

$$
\begin{aligned}
& \frac{1}{V} \iiint_{\mathscr{S}} T^{\circ}(r ; S) d V_{\boldsymbol{a}}=\frac{1}{V} \iiint_{\mathscr{S}} m^{\circ}(r, \boldsymbol{a} ; S) d V_{\boldsymbol{a}} \\
& \quad+\frac{1}{V} \iiint_{\mathscr{S}} N(r, \boldsymbol{a} ; S) d V_{\boldsymbol{a}}+\frac{1}{V} \iiint_{\mathscr{S}} H(r, \boldsymbol{a} ; S) d V_{\boldsymbol{a}} \\
& \quad+\frac{1}{V} \iiint_{\mathscr{S}} C(\boldsymbol{a} ; S) d V_{\boldsymbol{a}} .
\end{aligned}
$$

The integrand $T^{\circ}(r, S)$ of the integral in the left-hand member of (113) does not vary with $a$, and accordingly the value of this integral is $T^{\circ}(r ; S)$.

The first integral in the right-hand member of (113) is

$$
\begin{aligned}
& \frac{1}{V} \iiint_{\mathscr{S}} m^{\circ}(r, \boldsymbol{a} ; S) d V_{\boldsymbol{a}} \\
& =\frac{1}{V} \iiint_{\mathscr{S}}\left[\frac{1}{2 \pi} \int_{\partial_{A_{r}}(0)} \log \frac{1}{\chi(\boldsymbol{x}, \boldsymbol{a})} d \theta\right] d V_{\boldsymbol{a}} \\
& \quad=\frac{1}{2 \pi} \int_{\partial_{A_{\boldsymbol{r}}(0)}}\left[\frac{1}{V} \iiint_{\mathscr{S}} \log \frac{1}{\chi(\boldsymbol{x}, \boldsymbol{a})} d V_{\boldsymbol{a}}\right] d \theta,
\end{aligned}
$$

and here by geometric symmetry the inner integral in the last expression is the same for all $\boldsymbol{x} \in \mathscr{S}$. Accordingly, we can replace $\chi(\boldsymbol{x}, \boldsymbol{a})$ by $\chi(x(0,0), a)$ in (114). Except at $a=x(0,0)$, by (105) and (106), we have

$$
C(\boldsymbol{a} ; S)=\log \chi(x(0,0), \boldsymbol{a}) .
$$

Therefore the sum of the first and fourth integrals in the right-hand 
member of (113) is 0 .

The function $N(r, \boldsymbol{a} ; S)$ has value 0 for all $\boldsymbol{a}$ not on $S$. It therefore has value 0 everywhere except at most on a set of three-dimensional measure 0 on $\mathscr{S}$. Hence the second integral in the right-hand member of (113) has value 0 .

It follows, accordingly, that (113) can be written as

$$
T^{\circ}(r ; S)=\frac{1}{V} \int_{\infty} H(r, \boldsymbol{a} ; S) d V_{\boldsymbol{a}} .
$$

By (78), then, we have

$$
\begin{aligned}
T^{\circ}(r ; S) & =\frac{1}{V} \iiint_{\int}\left[\int_{0}^{r} \frac{h(\rho, \boldsymbol{a} ; S)}{\rho} d \rho\right] d V_{\boldsymbol{a}} \\
& =\int_{0}^{r}\left[\frac{1}{V} \iiint_{\mathscr{S}} h(\rho, \boldsymbol{a} ; S) d V_{\boldsymbol{a}}\right] \frac{d \rho}{\rho} \\
& =\int_{0}^{r} \mathscr{P}(\rho, S) \frac{d \rho}{\rho} .
\end{aligned}
$$

By (73), the function

$$
\mathscr{P}(\rho, S)=\frac{1}{V} \iiint_{\mathscr{S}} h(\rho, \boldsymbol{a} ; S) d V_{\boldsymbol{a}}
$$

is positive for $\rho>0$ and is a strictly increasing function of $\log \rho$. It can be given a quasi-geometric interpretation, as indicated in the discussion of $h(\rho \boldsymbol{a} ; S)$ in $\S 5$.

From (116) we obtain

$$
\frac{d T^{\circ}(r ; S)}{d \log r}=\mathscr{P}(r ; S) .
$$

Since $\mathscr{P}(r ; S)$ is positive for $r>0$ and is a strictly increasing function of $\log r$, and since $T^{\circ}(0 ; S)=0$, we therefore have the following result:

The function $T^{\circ}(r ; S)$ is positive for $r>0$ and is an increasing, strictly convex function of $\log r$.

It follows from (76), (81), and (101) that

$$
0 \leqq m(r, \infty ; S)<m^{\circ}(r, \infty, S)<m(r, \infty ; S)+\log 2^{\frac{1}{2}} .
$$

Therefore, by (90) and (108), the difference $T^{\circ}(r ; S)-T(r ; S)$ is a bounded function of $r$. Actually, it can be shown that, like $T^{\circ}(r ; S)$, the function $T(r ; S)$ is an increasing, strictly convex function of $\log r$.

The foregoing convexity properties are useful, in particular, in the study of problems of order and type [4] in the theory of meromorphic minimal surfaces. 


\section{REFERENCES}

1. L. V. Ahlfors, Beiträge der meromorphen Funktionen, 7. Congr. Math. Scand., Oslo, 1929.

2. E. F. Beckenbach, Meromorphic plane maps viewed from space (to appear).

3. E. F. Beckenbach and T. A. Cootz, The second fundamental theorem for meromorphic minimal surfaces (to appear).

4. E. F. Beckenbach and F. H. Eng, Rational minimal surfaces (to appear).

5. E. F. Beckenbach and J. W. Hahn, Triples of conjugate harmonic functions and minimal surfaces, Duke Math. J. 2 (1936), 698-704.

6. E. F. Beckenbach and G. A. Hutchison, Meromorphic minimal surfaces, Bull. Amer. Math. Soc. 68 (1962), 519-522.

7. E. F. Beckenbach and T. Radó, Subharmonic functions and minimal surfaces, Trans. Amer. Math. Soc. 35 (1933), 648-661.

8. L. R. Ford, Automorphic functions, McGraw-Hill Book Company, Inc., New York, 1929.

9. Einar Hille, Analytic function theory, Vol. 2, Ginn and Company, New York, 1962.

10. R. Nevanlinna, Zur Theorie der meromorphen Funktionen, Acta Math. 46 (1925), 1-99.

11. Barrett O'Neill, Elementary differential geometry, Academic Press, New York, 1966.

12. W. F. Osgood, Lehrbuch der Funktionentheorie, Vol. 1, B. G. Teubner, Leipzig, 1928.

13. Tibor Radó, On the problem of Plateau, Julius Springer Verlag, Berlin, 1933.

14. T. Shimizu, On the theory of meromorphic functions, Japanese J. Math. 6 (1929), 119-171.

15. M. Tsuji, Potential theory in modern function theory, Maruzen Company, Ltd., Tokyo, 1959.

Received September 8, 1967. The preparation of this paper was sponsored in part by the National Science Foundation Grant GP-5279.

UNIVERSity OF CALIFornia, LOS ANGELES 



\section{PACIFIC JOURNAL OF MATHEMATICS}

\section{EDITORS}

H. ROYDEN

Stanford University

Stanford, California

R. R Phelps

University of Washington

Seattle, Washington 98105
J. DUGUNDJI

Department of Mathematics

University of Southern California

Los Angeles, California 90007

RICHARD ARENS

University of California

Los Angeles, California 90024

\section{ASSOCIATE EDITORS}
E. F. BECKENBACH
B. H. NeumanN
F. WOLF
K. YosidA

\section{SUPPORTING INSTITUTIONS}

UNIVERSITY OF BRITISH COLUMBIA

CALIFORNIA INSTITUTE OF TECHNOLOGY

UNIVERSITY OF CALIFORNIA

MONTANA STATE UNIVERSITY

UNIVERSITY OF NEVADA

NEW MEXICO STATE UNIVERSITY

OREGON STATE UNIVERSITY

UNIVERSITY OF OREGON

OSAKA UNIVERSITY

UNIVERSITY OF SOUTHERN CALIFORNIA
STANFORD UNIVERSITY

UNIVERSITY OF TOKYO

UNIVERSITY OF UTAH

WASHINGTON STATE UNIVERSITY

UNIVERSITY OF WASHINGTON

AMERICAN MATHEMATICAL SOCIETY CHEVRON RESEARCH CORPORATION TRW SYSTEMS

NAVAL WEAPONS CENTER 


\section{Pacific Journal of Mathematics

Vol. 28, No. $1 \quad$ March, 1969

Patrick Robert Ahern, On the geometry of the unit ball in the space of real annihilating measures .............................. 1

Kirby Alan Baker, Equational classes of modular lattices ............. 9

E. F. Beckenbach and Gerald Andrew Hutchison, Meromorphic minimal surfaces ......................................... 17

Tae Ho Choe, Intrinsic topologies in a topological lattice ..............

John Bligh Conway, A theorem on sequential convergence of measures and

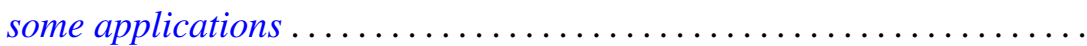

Roger Cuppens, On the decomposition of infinitely divisible probability laws without normal factor.

Lynn Harry Erbe, Nonoscillatory solutions of second order nonlinear

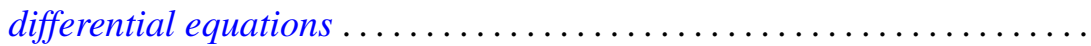

Burton I. Fein, The Schur index for projective representations of finite

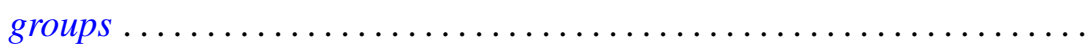

Stanley P. Gudder, A note on proposition observables............... 101

Kenneth Kapp, On Croisot's theory of decompositions ............... 105

Robert P. Kaufman, Gap series and an example to Malliavin's theorem . . . 117

E. J. McShane, Robert Breckenridge Warfield, Jr. and V. M. Warfield,

Invariant extensions of linear functionals, with applications to measures and stochastic processes ................

Marvin Victor Mielke, Rearrangement of spherical modifications ...

Akio Osada, On unicity of capacity functions ..............

Donald Steven Passman, Some 5/2 transitive permutation groups ...

Harold L. Peterson, Jr., Regular and irregular measures on groups and dyadic spaces...

Habib Salehi, On interpolation of $q$-variate stationary stochastic processes...

Michael Samuel Skaff, Vector valued Orlicz spaces generalized

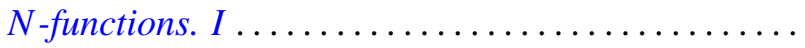

A. J. Ward, On $H$-equivalence of uniformities. II...........

Thomas Paul Whaley, Algebras satisfying the descending chain condition for subalgebras...

G. K. White, On subgroups of fixed index

Martin Michael Zuckerman, A unifying condition for implications among the axioms of choice for finite sets ................. 